\title{
A modified random walk door-to-door recruitment strategy for collecting social and biological data relating to mental health, substance use, addiction, and violence problems in a Canadian community
}

\author{
Andrea Flynn ${ }^{1}$, Paul F. Tremblay ${ }^{2}$, Jürgen Rehm ${ }^{3,4,5}$, and Samantha Wells ${ }^{1,4,6}$ \\ ${ }^{1}$ Centre for Addiction and Mental Health (CAMH), London, Canada \\ ${ }^{2}$ Department of Psychology, Western University, London, Canada \\ ${ }^{3}$ Centre for Addiction and Mental Health (CAMH), Toronto, Canada \\ ${ }^{4}$ Dalla Lana School of Public Health, University of Toronto, Toronto, Canada \\ ${ }^{5}$ Epidemiological Research Unit, Institute for Clinical Psychology and Psychotherapy, Dresden, Germany \\ ${ }^{6}$ Department of Epidemiology and Biostatistics, Western University, London, Canada
}

\begin{abstract}
Aims: To describe a modified "random walk" door-to-door recruitment strategy used to obtain a random community sample for participation in a study relating to mental health, substance use, addiction, and violence (MSAV) problems and involving the collection of both self-report and biological (hair and saliva) data. This paper describes study protocols, response rates for the study and for the provision of biological data, and possible further applications for this data collection method.
\end{abstract}

Design: A two-stage cluster sample was derived from the 2006 Canadian census sampling frame for a small Ontario community, based on the random selection of city blocks as the primary sampling units and households as the secondary sampling units.

Setting: A small city in Ontario, Canada.

Participants: A general population sample of 92 participants selected randomly from households using Kish tables.

Measures: A computerized questionnaire was administered to obtain self-report data on MSAV problems. Saliva was collected to study genetic vulnerabilities to MSAV problems, and hair was collected to examine stress levels (via the hormone cortisol) as they relate to MSAV problems.

Findings: The study showed a response rate of 50\% and a high rate of provision of biological samples (over 95\%).

Conclusions: Modified random walk methodologies involving face-to-face recruitment may represent a useful approach for obtaining general population samples for studies of MSAV problems, particularly those involving the collection of biological samples. Further studies are needed to assess whether this approach leads to better response rates and improved estimates compared to other survey methods used in research on substance use.

Population surveys are a key tool for epidemiologists, health planners, and policy makers, providing essential information about the prevalence and correlates of mental health and substance use problems that can be used to develop evidence-based public health programming and policy. In recent years, two important trends have emerged that hold implications for epidemiological research on substance use and addictions. First is the ongoing decline in survey response rates in numerous countries around the world, which can lead to bias in population estimates due to differences in respondent and non-respondent characteristics (Atrostic, Bates, Burt, \& Silberstein, 2001; de Heer, 1999; Steeh, 1981; Tolonen et al., 2006). Importantly, epidemiologic research focusing on health risk behaviors, including use of tobacco, alcohol, and illicit drugs, indicates that individuals who engage in these behaviors are often underrepresented among survey respondents (Burg, Allred, \& Sapp 1997; Cunradi, Moore, Killoran, \& Ames, 2005; Wild, Cunningham, \& Adlaf, 2001).

The second trend relates to the growing interest in biomarkers of health and the increasing tendency to collect biological data as part of epidemiological investigations

Correspondence: Andrea Flynn, Ph.D., Centre for Addiction and Mental Health, 100 Collip Circle, Suite 200, London, Ontario N6G 4X8, Canada. Telephone: 519858-5110. Fax 519-858-5133. E-mail: Andrea.Flynn@camh.ca

Financial support: Research for this article was supported by the Canadian Institutes of Health Research (CIHR), Emerging Team Grant (CBG-101926). The views expressed here do not necessarily reflect those of CIHR

Keywords: Random walk, cluster sampling, survey methods, biomarkers, mental health, substance use, addictions, violence 
(Morton, Cahill, \& Hartge, 2006). For example, a special section in the June 2010 issue of Alcoholism: Clinical and Experimental Research discussed advances (Bearer, Bailey, \& Hoek, 2010) and future prospects (Freeman \& Vrana, 2010) in alcohol biomarker research in clinical (Jatlow \& O’Malley, 2010) and applied settings (Litten, Bradley, \& Moss, 2010). The examination of biological data can be used to improve understanding of the biological mechanisms underlying substance use and abuse (e.g., see Müller et al., 2010). Moreover, biomarkers have been presented in the substance use literature as a way of validating self-report measures and improving estimates of substance use (e.g., Delaney-Black et al., 2010; Hahn et al., 2012). As Peterson (2010) points out, research on biomarkers linked to alcohol consumption has the potential to improve clinical assessment and treatment, and also stands to improve precision of measurement in research, going beyond the standard self-report measures that typically require participants to recall the number of drinks consumed over a specific time period. Advances in molecular techniques and increased recognition of the interactions between biological and social factors have led to the collection of both forms of data in studies of population health (Morton et al., 2006; Waite et al., 2010).

Given declines in study participation and the growing recognition that both social and biological data are needed to better understand substance use and addictions, innovative sampling and data collection strategies are required that maximize researchers' ability to obtain both types of data from representative population samples. In the present article we describe a "random walk" door-todoor recruitment strategy for randomly selecting a community sample for participation in a study on mental health, substance use, addiction, and violence (MSAV) problems. As part of a larger multidisciplinary program of research, we collected self-reported health information as well as biological specimens (saliva, for the extraction of DNA; hair, to analyze cortisol for the assessment of stress levels) to improve understanding of the independent and interactive effects of biological and social factors on MSAV problems (see Wells et al., 2011). The overarching goal of the present study was to develop a methodology for randomly selecting a general population sample and collecting both biological and social data as part of a community survey.

\section{Why new, innovative approaches to sampling are needed in health surveys}

Researchers relying on traditional survey methods, such as telephone and mail approaches, have increasingly struggled to generate adequate samples for epidemiological research purposes (Atrostic et al., 2001; de Heer, 1999; Steeh, 1981; Tolonen et al., 2006). Most developed countries still rely heavily on the telephone survey method to collect population health information, and the growing use of mobile phones, answering machines, voice mail, caller ID, and "do not call" lists are contributing to declines in response rates (Dillman, Eltinge, Groves, \& Little, 2002; Galea \& Tracy, 2007; Galesic, Tourangeau, \& Couper, 2006; Hartge, 1999; Kempf \& Remington 2007).
Telephone surveys relying on random digit dialing (RDD) may be particularly prone to noncoverage bias if only landlines are included in the sampling frame, since individuals who exclusively use mobile phones will be missed (Keeter, Kennedy, Clark, Tompson, \& Mokrzycki, 2007; Link, Battaglia, Frankel, Osborn, \& Mokdad, 2007; Tucker, Brick, \& Meekins, 2007). Young adults, for instance, tend to predominantly utilize mobile phones (Tucker et al., 2007), rendering this group particularly challenging to reach in telephone surveys relying on landline extensions. Likewise, internet surveys do not seem to hold much promise for augmenting response rates on population surveys (Link \& Mokdad, 2005; Lozar Manfreda, Bosnjak, Berzelak, Haas, \& Vehovar, 2008; Shih \& Fan, 2008); this is especially true when researchers are interested in collecting biological data. For example, Avendano, Mackenbach, \& Scherpenzeel (2010) evaluated the feasibility of collecting biomarkers (blood cholesterol, saliva cortisol, and waist circumference) through an internet survey and found response rates in the order of $15.0 \%$ (cholesterol) to $26.5 \%$ (waist circumference).

A household sampling procedure involving door-to-door recruitment and face-to-face contact may facilitate recruitment of survey participants by eliminating the need for accurate telephone listings and avoiding the noncoverage pitfall associated with RDD. In principle, a door-to-door recruitment approach involving in-person contact can allow for more convincing explanations of the importance of the research and assurances of confidentiality, both of which are particularly important for surveys about sensitive topics such as personal health (Aquilino, 1994). For example, in their recruitment of inner-city African-American women to a cancer screening intervention program, Blumenthal, Sung, Coates, Williams, and Liff (1995) found that in-person recruitment was superior to telephone recruitment.

Door-to-door recruitment approaches for epidemiological studies on substance use stand to be useful in diverse settings, including both developed and developing countries. Conducting high-quality surveys in developing countries is particularly challenging, as limitations on technology and resources impede the implementation of many advanced survey methodologies (e.g., computerassisted or web-based approaches) (Biemer \& Lyberg, 2003). Telephone and web-based surveys are usually impractical in developing countries because many people do not have a telephone or access to a computer. Many regions lack the documented population/household information needed for generating sampling frames (Biemer \& Lyberg, 2003), while in other areas, conflict or instability complicate survey implementation (Haer \& Becher, 2012).

Door-to-door recruitment may also have particular applicability to epidemiological studies involving the collection of biological data. With a few exceptions (e.g., Avendano et al., 2010; Jaszczak, Lundeen, \& Smith, 2009), participation rates in epidemiologic surveys that include a biological component are not well known, partly due to poor reporting practices in the epidemiological literature 
(see Morton et al., 2006). Moreover, little is known about how response rates associated with biological sample provision compare to other survey components. Few studies have combined the collection of both biological and social data on a large scale, and little research has been conducted on the most effective methods of collecting biological specimens as part of household surveys. Overall, researchers suggest that better field methods for collecting biological data in epidemiological surveys are needed (Jaszczak et al., 2009) and argue the need to report response rates separately for biological and non-biological components (Morton et al., 2006).

\section{The "Random Walk" Method}

The random walk approach is a simplified cluster sampling method developed by the World Health Organization (WHO) and originally implemented in the Expanded Programme on Immunization (EPI) (see Bennett, Woods, Liyanage, \& Smith, 1991; Henderson \& Sundaresan, 1982; Lemeshow \& Robinson, 1985). In this approach, a population is divided into a specified number of geographic “clusters" (e.g., villages, neighbourhoods, etc.) of a known or estimable population size (Lemeshow \& Robinson, 1985). Clusters are randomly selected with probability proportionate to size (PPS) (i.e., larger clusters are more likely to be selected), and then the desired number of households per cluster are selected (Bennett et al., 1991; Lemeshow \& Robinson, 1985). The method for randomly selecting households depends on the size, density, and living arrangements of the population within the cluster. In small villages, full enumeration of households, followed by random sampling, is often possible. In scattered populations, randomly selecting a direction to walk (e.g., by spinning a bottle), selecting a random starting point, and sampling contiguous households is common (Bennett et al., 1991; Lemeshow \& Robinson, 1985). Random selection in urban settings can be more challenging, given the larger sampling frame and complex household types (e.g., apartment buildings). In such settings, a common approach is to divide the geographic area of interest into zones, randomly select a zone, and randomly select a starting point within that zone. However, specific strategies must be devised for selecting households in multiple-dwelling units (Lemeshow \& Robinson, 1985).

The traditional EPI methodology has important limitations. Selecting geographically contiguous households, which may share key factors (e.g., environmental stressors), could affect survey findings (Lemeshow \& Robinson, 1985). The EPI method also does not include an approach for revisiting households in cases of non-response; potential bias may result if individuals who are not at home share characteristics that differ from those who are at home (Lemeshow \& Robinson, 1985).

Various efforts have been made to extend the EPI approach in diverse settings. Milligan, Njie, and Bennett (2004) favor compact segment sampling, a self-weighting procedure involving the selection of clusters with PPS using census data (see also Turner, Magnani, \& Shuaib, 1996). Technologies such as Global Positioning System (GPS) (used to study vaccination coverage in Niger; Grais,
Rose, \& Guthmann, 2007) and Geographic Information Systems/Google Earth imagery (used for a mortality survey in Iraq; Galway et al., 2012) have been advanced as ways of improving the EPI approach. Various topics have been studied using modified random walk methodologies, including adolescent social networks and HIV transmission in the U.S. (McGrady et al., 1995), and mental health in the Balkans (Priebe et al., 2010) and Germany (Mundt et al., 2012), although response rates have not always been higher than telephone survey response rates. In Mundt et al.'s study (2012), over half of the original sample did not respond, while an additional 25\% were living in another community at the time, leading to a final response rate of $48.5 \%$.

Overall, the literature on random walk sampling suggests that this methodology shows promise for studying a variety of health-related topics, but that it can be refined through use of web-based maps (e.g., Google Maps) and other related technologies. The present paper describes an application of a modified random walk approach to gather social and biological data in a small Canadian community. We describe the study protocols and provide response rates for the study and for the provision of biological data. Implications of the preliminary findings for research on substance use, and suggestions for future research, are provided.

\section{Method}

This pilot study was conducted as part of Researching Health in Ontario Communities (RHOC), a multidisciplinary study funded by the Canadian Institutes of Health Research (CBG-101926) that uses a mobile research lab to collect social, epidemiological and biological data in communities across Ontario (see Wells et al., 2011). The random walk study was implemented in Port Colborne, a small city in Southern Ontario with a population of 18,599 as of the 2006 Canadian Census. This study was approved by the Research Ethics Board at the Centre for Addiction and Mental Health (CAMH).

\section{Research Design}

A two-stage cluster sample approach involving the random selection of blocks (primary sampling units [PSU]) within census tracts, households (secondary sampling units [SSU]) within blocks, and individuals (elementary units) within households was employed. The sampling frame was generated from 2006 Canadian Census data for Port Colborne. In 2006, Port Colborne was characterized by five tracts, ranging from 3,193 to 4,856 residents. Across these tracts were 427 blocks with a mean population of 43.56 and a mean number of households of 19.95. The total number of private dwellings was 8,519.

The sampling frame was comprised of 200 households. This sampling frame was deemed sufficiently large for the purpose of developing protocols. First, a sample of census blocks (clusters) was selected with PPS. CSurvey 2.0 (Farid \& Frerichs, 2007) was used to select 20 blocks using 
PPS with replacement (i.e., a block could be selected more than once; in the present sample, three blocks were randomly selected twice). These blocks ranged in size from 11 to 235 households. "Households" were defined as places of residence identified by an address number, a mailbox, and/or an apartment number. A sample of 10 households was collected from 17 of the 20 randomly selected blocks, and a sample of 20 households was collected from the three blocks that were randomly selected twice. Prior to data collection, maps for the census tracts and for each randomly selected block were prepared. The maps were used to randomly select one of the four corners of a block; the household closest to the selected corner was identified as the starting point. Next, a random number ranging from zero to the total number of households in the block minus one was selected. Then, using aerial maps, the number of households corresponding to the selected random number was counted in a clockwise direction starting with the household closest to the selected corner. The field workers were instructed to proceed from the starting point in a clockwise direction and were provided with the specific number of households to be skipped in each block in order to ensure objectivity in household selection.

\section{Participant Recruitment and Data Collection}

Each randomly selected household was contacted up to six times by a combination of methods - via letter (dropped off by staff), in person, or by phone - to request their voluntary participation. Participation involved visiting the CAMH mobile research lab, parked in a convenient location in the community, to complete a self-administered questionnaire and provide biological samples. During the first stage of recruitment, staff randomly selected 200 households and dropped off an advance letter and a flyer about the study. Field workers travelled in male-female pairs to ensure their safety. They wore CAMH photo identification badges and carried study investigators' business cards and additional project literature. On the second household visit, the field workers knocked on the doors of the randomly selected homes. If contact was not made, a second letter was left. Subsequent visits were made as needed, such that each household was visited in person up to three times, not including the first advance letter drop-off. Times and days of the week for these visits were systematically varied to maximize the chance of contact. If contact was not made, the staff tried to locate telephone numbers using available listings (Canada411.ca). Additional recruitment attempts were made by telephone, with a total of three callbacks at varying times of day and days of the week. Households for which telephone numbers could not be found were revisited in person up to six times.

When contact was made, the field staff obtained permission to randomly select an adult for participation. If the resident reached was the only person living in the household and was 18 or older, they were automatically selected. Otherwise, the researchers used Kish tables (Kish, 1949) to randomly select an adult within the household. Informed consent was obtained at the lab prior to data collection. Participants were compensated in gift cards; they received
\$25 for completing the questionnaire and \$25 for providing one or both of the biological samples.

Efforts were also made to raise the profile of the study in the community to encourage participation. First, the RHOC project's Community Advisory Committee, consisting of representatives from local and regional health organizations (see Wells et al., 2011), raised awareness of the project through their networks. Second, the research team delivered a televised presentation to city council and held meetings with community stakeholders (e.g., police). Third, the study was promoted in community newspapers and local news channels.

\section{Results}

Figure 1 summarizes participation and refusals. Of the initial 200 households, 11 were ineligible because they were vacant or belonged to non-Canadian citizens (e.g., summer homes owned by U.S. residents). Interviewers were able to make contact with 186 of the remaining 189 households. Approximately $47 \%$ of contacted residents were female and $53 \%$ were male.

As shown in Figure 1, in 146 (78.5\%) of the 186 contacted households, either the residents agreed to complete Kish tables or there was only one resident in the household (who was automatically selected). A larger proportion of men (84.4\%) than women (76.2\%) agreed to the selection process $\left(\chi_{(1)}^{2}=1.92\right.$, not significant). Of the 146 residents selected for participation, 83 (56.8\%) were men and 63 (43.2\%) were women. As shown in Table 1, the selection process using Kish tables led to a higher proportion of men (56.8\%) than exists in the composition of the 146 households involved in the selection process (53.9\%). This discrepancy can be partly accounted for by gender differences in the number of single resident households.

Of the 146 selected persons, 95 agreed to participate at the time of contact (54 of the 83 men [65.1\%] and 41 of 63 women [65.1\%] who were selected). After multiple attempts, the field workers were unable to reach nine of the 146 selected persons. Three of the 95 people who initially agreed to participate ultimately did not take part in the study. The final sample $(n=92)$ consisted of 52 men (56.5\%) and 40 women (43.5\%).

\section{Response Rate}

The final response rate, defined as the number of participants $(n=92)$ divided by the number of contacted households ( $n=186$ ), was $49.5 \%$. Although the intent of the first field visit was only to drop off the advance letter, field workers contacted residents in $54.3 \%$ of the households during this first visit (see Figure 2). By the second and third recruitment visits, contact had been made in $72.6 \%$ and $80.1 \%$ of the 186 households, respectively. Additional visits and telephone calls were made to reach the remaining households. Non-contact occurred for only three eligible households. Figure 2 shows a similar cumulative distribution for participation appointments (i.e., 
Figure 1

Participation and refusals at different stages of recruitment

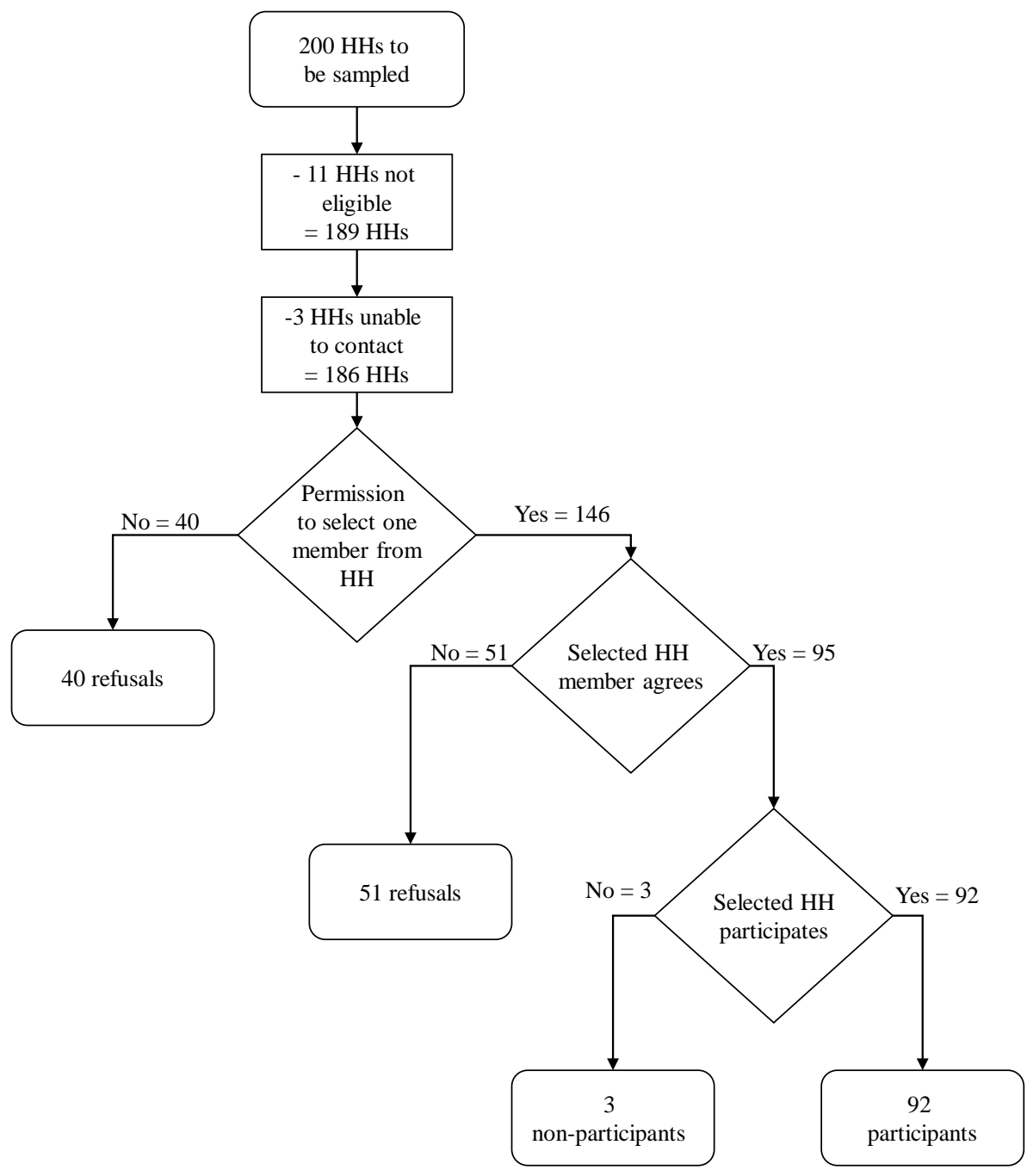

Table 1

Distribution by gender

\begin{tabular}{lcccc}
\hline & Gender \% based on & Gender \% based on \\
Group & $152(53.9 \%)$ & $83(56.8 \%)$ & $\begin{array}{c}\text { Gender \% based on } \\
\text { Kish table with } \\
\text { exclusion of single } \\
\text { resident households }\end{array}$ \\
\hline Men $n(\%)$ & $130(46.1 \%)$ & $63(43.2 \%)$ & $27(69.2 \%)$ & $56(52.3 \%)$ \\
Female $n(\%)$ & 282 & 146 & $12(30.8 \%)$ & $51(47.7 \%)$ \\
Total $N$ & & & 39 & 107 \\
\hline
\end{tabular}


Figure 2

Cumulative percentage of households in which contact was established or appointment was set as a function of the visit



when a specific time for participants to come to the mobile lab for survey completion was set). After the third visit, $63 \%$, or 58 of the 92 appointments, were set. Additional contact attempts were required to book the other $37 \%$ of participation appointments.

\section{Provision of Biological Samples}

Of the 92 study participants, 88 (95.6\%) provided a saliva sample. Participants were eligible to provide hair samples only if their hair was at least $3 \mathrm{~cm}$ long, the minimum length needed for analysis. The individuals who were ineligible to provide hair samples were primarily men. Seventy-five participants met the eligibility criterion for hair collection; of these, seventy-four provided hair samples (98.7\%).

\section{Discussion}

This paper describes a modified "random walk" door-todoor recruitment strategy used to obtain a random community sample for participation in a study involving the collection of both self-report and biological data (hair and saliva) relating to mental health, substance use, addiction, and violence (MSAV) problems. The study demonstrated a relatively good response rate of $49.5 \%$, which is similar to the response rate of $48.5 \%$ obtained in a recent random walk study on mental health conducted in Berlin (Mundt et al., 2012). The study also demonstrated an exceptionally good cooperation rate on the provision of biological samples (96\% for saliva, almost $99 \%$ of eligible participants for hair). These findings add to growing evidence that participants are willing to provide saliva samples for genetic research (Hansen, Simonsen, Nielsen, \& Hundrup, 2007) and demonstrate the feasibility of integrating the collection of biological data into epidemiological surveys on MSAV problems.

In-person recruitment using door-to-door approaches may be especially advantageous when it is deemed important to explain to potential participants the significance of the research or the details of the project, such as how biological data will be collected and used. In the present study, trained research staff gained the trust of potential participants by showing their staff identification, providing relevant literature when requested, and answering specific questions about the project. These strategies are often not possible using other survey methods.

Non-contact is a key factor affecting declining response rates in epidemiologic studies (Galea \& Tracy, 2007), with, for example, non-contact in telephone surveys rising due to the use of cell phones, caller ID and do-not-call lists (Dillman et al., 2002; Galea \& Tracy, 2007; Galesic et al., 2006; Hartge, 1999; Kempf \& Remington, 2007). In contrast, the present approach maximized contact with potential participants. After six contact attempts, noncontact occurred in only three of 189 (1.6\%) eligible households. Thus, the potential bias associated with noncontact was almost nonexistent in the present study.

The present study offers several valuable modifications to the traditional random walk approach that may be applicable across diverse settings. The household selection process for the study was completed using current 
technology (aerial photos from Google Maps), thus eliminating subjectivity. Taking advantage of technological innovations may be especially helpful when implementing the random walk approach in complex urban settings. Another key modification to the random walk approach was that participants were required to visit the mobile research lab for data collection, rather than having data collected in their homes. While potential participants may be reluctant to visit large study sites such as universities or hospitals (Djuric et al., 2008), they appear to be willing to participate when small clinics, such as mobile labs, are available in their communities. With this approach, staff safety was also maximized, since they did not have to enter participants' homes. Participants could more easily keep their involvement confidential from other household members, if desired. Use of the lab also meant that all necessary research materials and data collection tools did not need to be transported into the field. Combining a door-to-door recruitment strategy with data collection in a secure, neutral location may thus be an important way of augmenting response rates, enhancing standardization of data collection, and facilitating biological sample collection. Notably, however, securing the technology and resources needed to use the mobile lab may not be feasible in regions where research resources are scarce.

The study had a number of limitations which have implications for application of this method in future research. Forty of the 186 (21.5\%) eligible households refused participation from the outset of the study. This placed a ceiling of $78.5 \%$ on the response rate and meant that demographic data on the eligible household member was not obtained, affecting analyses of contact and refusal rates. Future research could implement strategies to reduce this initial refusal rate. In this study, household members who assisted in the completion of Kish tables but were not randomly selected were not compensated for their time. A small incentive provided at the time of selection could decrease refusals at this stage, to the possible end of raising the final response rate. An important question for consideration is whether asking for the provision of biological samples was a reason for participant refusal. Unfortunately, this information was not collected in the present study. Therefore, in future research it will be necessary to assess the extent to which a request for biological data is a deterrent for potential participants.

Another limitation is the cost associated with the fieldwork. We have estimated this cost at roughly $\$ 300$ per participant, assuming a response rate of $50 \%$ in a sample of 200 participants, excluding compensation to study participants. Travelling costs for door-to-door recruitment were minimal in our present study due to the fact that sampling occurred in a small community. This cost could increase substantially if interviewers had to travel longer distances between clusters in large cities or rural areas. As noted above, interviewers were always sent in pairs (male and female) for their security, which doubled the cost of human resources for the door-to-door recruitment component but was deemed necessary to ensure staff safety. Notably, costs of the study would diminish as the sample size increases, because some resources and tasks, such as preparation of maps and travelling to selected blocks, are largely independent of the number of participants. Another way to decrease cost would be to reduce the number of repeated visits to households. In the present study, three visits were required to make first contact with $80 \%$ of the sample. After three visits, only $63 \%$ (58 of the 92 households) of selected individuals had set appointments for participation. This meant that additional visits and telephone calls were required to reach the remaining $37 \%$ of households. Therefore, any reductions in the number of visits would reduce the response rate dramatically. Overall, while approaches to cost cutting may be employed, they must be weighed against potential loss in data quality.

\section{Conclusion}

Future research using the random walk method can build on the present study to investigate ways of improving on the current response rate of $50 \%$. Strategies for converting refusals, including offering incentives at various stages in the recruitment process and providing alternative ways of participating, could be investigated. Future research could also investigate the impact of requesting biological samples on response rates and the effect of stigma on participation (i.e., whether people may have refused to participate because of the study's focus on mental health, substance use, and violence) (see Pescosolido et al., 2010). Such efforts would allow for further refinement and adaptation of the proposed methodology to suit the survey and community under investigation.

As Morton et al. (2006) point out, detailed information regarding recruitment and participation is critical to advancing the epidemiological literature and expanding researchers' capacity to improve survey methods. Future research is needed that directly compares sampling strategies in terms of response rates, sample representativeness, and costs for multiple recruitment strategies and data collection methods (e.g., telephone, mail, door-to-door) within the same community or comparable communities, using sufficiently large samples. This research could also examine several variations on the methods (e.g., door-to-door recruitment with data collection occurring in participants' homes vs. door-to-door recruitment with data collection occurring in a centralized data collection location, such as a mobile lab) in order to capture the impact of different components of the method on response rates. Such a study would permit rigorous evaluation of the relative merits and weakness of different approaches for studying substance use and abuse and other health-related topics, including an assessment of whether provision of biological samples would vary as a function of the method employed.

Potential applications of the random walk methodology in future research are numerous. Given that most participants were willing to provide biological data in the current study, the random walk approach for collecting both social and biological data may be useful for examining biological mechanisms underlying substance use and abuse (e.g., see Müller et al., 2010) as well as interactions between 
biological and social factors as they relate to mental health and substance use problems (Morton et al., 2006; Waite et al., 2010). As discussed earlier in this paper, the random walk methodology may be particularly valuable in developing countries, where population and telephone listings are unavailable but general population samples and collection of biological data are desired. Applying a random walk methodology combined with collection of biomarkers would allow for objective population-level estimates of alcohol use and abuse, which are crucial for the development of evidence-based policy and programming. In developing countries, for example, studies have revealed the value of collecting biomarkers of alcohol use in settings where traditional measures of alcohol consumption are hindered by various obstacles (e.g., the common use of home-distilled alcohol and sharing of alcohol in social drinking settings - see Hahn et al., 2012). Furthermore, it is reasonable to posit that the random walk methodology could serve as a mechanism for dovetailing research and alcohol education and abuse prevention campaigns, including, for instance, population education on the risks of alcohol use and provision of information about available services in the community. Such an approach mirrors the principles of the original EPI, involving assessment of immunization coverage in combination with provision of vaccines. The random walk methodology also holds relevance for urban settings, with potential to help researchers overcome some of the limitations of telephone-based surveys on alcohol use (see Shield \& Rehm, 2012) by enhancing the likelihood of contact and by allowing for more detailed collection of information about participation and refusals. Overall, further research using this method can help clarify its potential applications and refine the approach for use in diverse settings.

\section{Acknowledgements}

Research for this article was supported by the Canadian Institutes of Health Research (CIHR), Emerging Team Grant (CBG-101926). The views expressed here do not necessarily reflect those of CIHR. We are grateful to the field staff involved in participant recruitment and data collection and to Sue Steinback for assistance with references and formatting.

\section{References}

Aquilino, W. S. (1994). Interview mode effects in surveys of drug and alcohol use. Public Opinion Quarterly, 58, 210-240.

Atrostic, B. K., Bates, N., Burt, G., \& Silberstein, A. (2001). Nonresponse in U.S. government household surveys: Consistent measures, recent trends, and new insights. Journal of Official Statistics, 17, 209-226.

Avendano, M., Mackenbach, J., \& Scherpenzeel, A. (2010). Can biomarkers be collected in an Internet survey? A pilot study in the LISS panel. In M. Das, P. Ester, \& L. Kaczmirek (Eds.), Social and behavioral research and the internet: Advances in applied methods and research strategies. Oxford, UK: Taylor and Francis.

Bearer, C., Bailey, S. M., \& Hoek, J. B. (2010). Advancing alcohol biomarkers research. Alcoholism: Clinical and Experimental Research, 34, 941945.

Bennett, S., Woods, T., Liyanage, W. M., \& Smith, D. L. (1991). A simplified general method for clustersample surveys of health in developing countries. World Health Statistics Quarterly, 44, 98-106.

Biemer, P. P., \& Lyberg, L. E. (2003). Introduction to Survey Quality. Hoboken, NJ, United States: John Wiley \& Sons Inc.

Blumenthal, D. S., Sung, J., Coates, R., Williams, J., \& Liff, J. (1995). Mounting research addressing issues of race/ethnicity in health care: Recruitment and retention of subjects for a longitudinal cancer prevention study in an innercity black community. Health Services Research, 30, 197-205.

Burg, J. A., Allred, S. L., Sapp, J. H. 2nd. (1997). The potential for bias due to attrition in the National Exposure Registry: An examination of reasons for nonresponse, nonrespondent characteristics, and the response rate. Toxicology and Industrial Health, 13, 1-13.

Cunradi, C.B., Moore, R., Killoran, M., Ames, G. (2005). Survey nonresponse bias among young adults: The role of alcohol, tobacco, and drugs. Substance Use \& Misuse, 40, 171-185.

de Heer, W. (1999). International response trends: Results of an international survey. Journal of Official Statistics, 15, 129-142.

Delaney-Black, V., Chiodo, L. M., Hannigan, J. H., Greenwald, M. K., Janisse, J., Patterson, G., . . . Sokol, R. J. (2010). Just say "I don't”: Lack of concordance between teen report and biological measures of drug use. Pediatrics, 126, 887-893. doi:10.1542/peds.2009-3059

Dillman, D. A., Eltinge, J. L., Groves, R. M., \& Little, R. J. A. (2002). Survey nonresponse in design, data collection, and analysis. In R. M. Groves, D. A. Dillman, J. S. Eltinge, \& R. J. A. Little (Eds.), Survey nonresponse (pp. 3-26). New York, New York, United States: John Wiley.

Djuric, Z., Bird, C. E., Furumoto-Dawson, A., Rauscher, G. H., Ruffin IV, M. T., Stowe, R. P., ... Masi, C. M. (2008). Biomarkers of psychological stress in health disparities research. The Open Biomarkers Journal, 1, 7-19.

Farid, M. N., \& Frerichs, R. R. (2007). CSurvey Version 2.0 (2007). University of California. http://www.ph.ucla.edu/epi/csurvey.html.

Freeman, W. M., \& Vrana, K. E. (2010). Future prospects for biomarkers of alcohol consumption and alcohol-induced disorders. Alcoholism: Clinical and Experimental Research, 34, 946-954.

Galea, S., \& Tracy, M. (2007). Participation rates in epidemiologic studies. Annals of Epidemiology, 17, 643-653. 
Galesic, M., Tourangeau, R., \& Couper, M. P. (2006). Complementing random-digit-dial telephone surveys with other approaches to collecting sensitive data. American Journal of Preventive Medicine, 31, 437-443.

Galway, L. P., Bell, N., Sae, A. S., Hagopian, A., Burnham, G., Flaxman, A., ... Takaro, T. K. (2012). A two-stage cluster sampling method using gridded population data, a GIS, and Google Earth $^{\mathrm{TM}}$ imagery in a population-based mortality survey in Iraq. International Journal of Health Geographics, 11. doi:10.1186/1476-072X-11-12.

Grais, R. F., Rose, A. M. C., \& Guthmann, J.-P. (2007). Don't spin the pen: Two alternative methods for second-stage sampling in urban cluster surveys. Emerging Themes in Epidemiology, 4. doi: 10.1186/1742-7622-4-8.

Haer, R., \& Becher, I. (2012). A methodological note on quantitative field research in conflict zones: Get your hands dirty. International Journal of Social Research Methodology, 15, 1-13.

Hahn, J. A., Dobkin, L. M., Mayanja, B., Emenyonu, N. I., Kigozi, I. M., Shiboski, S., ... Wurst, F. M. (2012). Phosphatidylethanol (PEth) as a biomarker of alcohol consumption in HIVpositive patients in Sub-Saharan Africa. Alcoholism: Clinical and Experimental Research, 36(5), 854-862.

Hansen, T. V., Simonsen, M. K., Nielsen, F. C., \& Hundrup, Y. A. (2007). Collection of blood, saliva, and buccal cell samples in a pilot study on the Danish nurse cohort: Comparison of the response rate and quality of genomic DNA. Cancer Epidemiology, Biomarkers \& Prevention, 16, 2072-2076. doi: 10.1158/1055-9965.EPI-070611

Hartge, P. (1999). Raising response rates: Getting to yes. Epidemiology, 10, 105-107.

Henderson, R. H., \& Sundaresan, T. (1982). Cluster sampling to assess immunization coverage: A review of experience with a simplified sampling method. Bulletin of the World Health Organization, 60, 253-260.

Jaszczak, A., Lundeen, K., \& Smith, S. (2009). Using nonmedically trained interviewers to collect biomeasures in a national in-home survey. Field Methods, 21, 26-48.

Jatlow, P., \& O’Malley, S. S. (2010). Clinical (nonforensic) application of ethyl glucuronide measurement: Are we ready? Alcoholism: Clinical and Experimental Research, 34, 968975.

Keeter, S., Kennedy, C., Clark, A., Tompson, T., \& Mokrzycki, M. (2007). What's missing from national landline RDD surveys? The impact of the growing cell-only population. Public Opinion Quarterly, 71, 772-792.

Kempf, A. M., \& Remington, P. L. (2007). New challenges for telephone survey research in the twenty-first century. Annual Review of Public Health, 28, 113-126.
Kish, L. (1949). A procedure for objective respondent selection within the household. Journal of the American Statistical Association, 44, 380-387.

Lemeshow, S., \& Robinson, D. (1985). Surveys to measure programme coverage and impact: A review of the methodology used by the expanded programme on immunization. World Health Statistics Quarterly, 38, 65-75.

Link, M. W., Battaglia, M. P., Frankel, M. R., Osborn, L., \& Mokdad, A. H. (2007). Reaching the U.S. cell phone generation: Comparison of cell phone survey results with an ongoing landline telephone survey. Public Opinion Quarterly, 71, 814-839.

Link, M. W., \& Mokdad, A. H. (2005). Alternative modes for health surveillance surveys: An experiment with web, mail, and telephone. Epidemiology, 16, 701-704.

Litten, R. Z., Bradley, A. M., \& Moss, H. B. (2010). Alcohol biomarkers in applied settings: Recent advances and future research opportunities. Alcoholism: Clinical and Experimental Research, 34, 955-967.

Lozar Manfreda, K., Bosnjak, M., Berzelak, J., Haas, I., \& Vehovar, V. (2008). Web surveys versus other survey modes: a meta-analysis comparing response rates. International Journal of Market Research, 50, 79-104.

McGrady, G. A., Marrow, C., Myers, G., Daniels, M., Vera, M., Mueller, C., ... Lovely, R. (1995). A note on implementation of a random-walk design to study adolescent social networks. Social Networks, 17, 251-255.

Milligan, P., Njie, A., \& Bennett, S. (2004). Comparison of two cluster sampling methods for health surveys in developing countries. International Journal of Epidemiology, 33, 469-476.

Morton, L. M., Cahill, J., \& Hartge, P. (2006). Reporting participation in epidemiologic studies: A survey of practice. American Journal of Epidemiology, 163, 197-203.

Müller, D. J., Likhodi, O., Heinz, A. (2010). Neural markers of genetic vulnerability to drug addiction. Current Topics in Behavioral Neurosciences, 3, 277-299.

Mundt, A. P., Aichberger, M. C., Kliewe, T., Ignatyev, Y., Yayla, S., Heimann, H., ... Ströhle, A. (2012). Random sampling for a mental health survey in a deprived multi-ethnic area of Berlin. Community Mental Health Journal, 48, 792-797.

Pescosolido, B. A., Martin, J. K., Long, J. S., Medina, T. R., Phelan, J. C., \& Link, B. G. (2010). “A disease like any other”? A decade of change in public reactions to schizophrenia, depression, and alcohol dependence. American Journal of Psychiatry, 167, 1321-1330.

Peterson, K. (2010). Biomarkers for alcohol use and abuse-A summary. Alcohol: Clinical Experimental Research, 34(6), 955-967.

Picavet, H. S. J. (2001). National health surveys by mail or home interview: Effects on response. Journal of Epidemiology \& Community Health, 55, 408413. 
Priebe, S., Bogic, M., Ajdukovic, D., Franciskovic, T., Galeazzi, G. M., Kucukalic, A., ... Schützwohl, M. (2010). Mental disorders following war in the Balkans: A study in 5 countries. Archives of General Psychiatry, 67, 518-528.

Shield, K. D., \& Rehm, J. (2012). Difficulties with telephone-based surveys on alcohol consumption in high-income countries: The Canadian example. International Journal of Methods in Psychiatric Research, 21, 17-28.

Shih, T-H., \& Fan, X. (2008). Comparing response rates from web and mail surveys: A meta-analysis. Field Methods, 20, 249-271.

Steeh, C. G. (1981). Trends in nonresponse rates, 19521979. Public Opinion Quarterly, 45, 40-57.

Tolonen, H., Helakorpi, S., Talala, K., Helasoja, V., Martelin, T., \& Prättälä, R. (2006). 25-year trends and socio-demographic differences in response rates: Finnish adult health behaviour survey. European Journal of Epidemiology, 21, 409-415.

Tucker, C., Brick, J. M., \& Meekins, B. (2007). Household telephone service and usage patterns in the United States in 2004: Implications for telephone samples. Public Opinion Quarterly, 71, 3-22.

Turner, A. G., Magnani, R. J., \& Shuaib, M. (1996). A not quite as quick but much cleaner alternative to the Expanded Programme on Immunization (EPI) Cluster Survey design. International Journal of Epidemiology, 25, 198-203.

Waite, L. J., Laumann, E. O., Levinson, W., Tessler Lindau, S., McClintock, M. K., O'Muircheartaigh, C. A., ... Schumm, L. P. (2010). National Social Life, Health, and Aging Project (NSHAP). ICPSR20541-v5. Ann Arbor, MI, United States: Inter-university Consortium for Political and Social Research. doi:10.3886/ICPSR20541.v5

Wells, S., Flynn, A., Graham, K., Rehm, J., Cairney, J., Kates, N., ... Verjee, Z (2011). Using a mobile laboratory to study mental health, addictions, and violence: A research plan. Challenges, 2, 1-18.

Wild, T. C., Cunningham, J., \& Adlaf, E. (2001). Nonresponse in a follow-up to a representative telephone survey of adult drinkers. Journal of Studies on Alcohol, 62, 257-261. 